\section{Tumores del estroma gastrointestinal: revisión y manejo multidisciplinario}

Gastrointestinal stromal tumors: multidisciplinary review and management

Wendy Liceth Villafuerte Quimiz

Médico, Hospital Miguel Hilario Alcívar, Bahía de

Caráquez, Ecuador wendyvq08@gmail.com,

https://orcid.org/0000-0002-4927-0373

Ingrid Verónica Ostaiza Véliz

Médico, Centro de Salud El Cisne II Tipo C,

Guayaquil, Ecuador, ingridmd28@hotmail.com,

https://orcid.org/0000-0002-7998-1264

Lissette Nicole Williams Vargas

Médico, YANAPAY S.A, Guayaquil, Ecuador,

lissetenwv1991@gmail.com,

https://orcid.org/0000-0002-6716-5446

Xavier Palomeque Salazar

Especialista en traumatología y ortopedia, Hospital

General del Norte de Guayaquil Los Ceibos,

Guayaquil, Ecuador,

xavierpalomeque@gmail.com,

https://orcid.org/0000-0002-3603-0442

Guayaquil - Ecuador

http://www.jah-journal.com/index.php/jah

Journal of American health

Enero - Marzo vol. 4. Num. 1-2021

Esta obra está bajo una Licencia Creative Commons

Atribución-NoComercial-CompartirIgual 4.0 Internacional.

RECIBIDO: 8 DE FEBRERO 2020

ACEPTADO: 2 DE DICIEMBRE 2020

PUBLICADO: 4 DE ENERO 2021

\section{RESUMEN}

Los tumores del estroma gastrointestinal (GIST) comprenden < 1\% de todos los tumores gastrointestinales (GI), pero los GIST son los tumores mesenquimales más comunes del tracto $\mathrm{Gl}$. Se han observado cambios drásticos en la práctica clínica en la última década. A pesar de un progreso considerable en el diagnóstico y tratamiento de los GIST, se estima que aproximadamente la mitad de todos los pacientes experimentan recurrencia. El objetivo del presente estudio es proporcionar una revisión actualizada de los conceptos básico sobre el tumor y la gestión general, además de los desarrollos recientes. Se analizaron artículos con menos de diez años de publicación, incluyendo artículos de revisión, metaanálisis y reportes de casos; no hubo restricción de idioma para la selección de los artículos. Conclusión: Esta revisión de la literatura aclara algunos aspectos sobre epidemiología, fisiopatología, inmunohistoquímica, tratamiento quirúrgico y terapia coadyuvante. Los GIST son tumores poco frecuentes que representan un pequeño porcentaje de neoplasias gastrointestinales y suelen ser positivos para CD117 y DOG-1. La resección quirúrgica laparoscópica es el estándar de oro para el tratamiento de GIST. Las metástasis son muy comunes y se pueden ver en el hígado, el mesenterio y el epiplón. Se recomienda el tratamiento coadyuvante con inhibidores de la tirosina quinasa en aquellos pacientes que tienen GIST primarios de alto riesgo.

PALABRAS CLAVE: CD117, tratamiento, pronóstico, tumores del estroma gastrointestinal, Inhibidores de tirosina quinasa. 


\section{ABSTRACT}

Gastrointestinal stromal tumors (GIST) comprise $<1 \%$ of all gastrointestinal (GI) tumors, but GISTs are the most common mesenchymal tumors of the GI tract. Drastic changes in clinical practice have been observed in the last decade. Despite considerable progress in the diagnosis and treatment of GISTs, it is estimated that approximately half of all patients experience recurrence. The aim of the present study is to provide an up-to-date review of basic concepts about tumor and general management, in addition to recent developments. Articles with less than ten years of publication were analyzed, including review articles, meta-analysis and case reports; There was no language restriction for the selection of articles. Conclusion: This literature review clarifies some aspects of epidemiology, pathophysiology, immunohistochemistry, surgical treatment and adjuvant therapy. GISTs are rare tumors that represent a small percentage of gastrointestinal neoplasms and are usually positive for CD117 and DOG-1. Laparoscopic surgical resection is the gold standard for the treatment of GIST. Metastases are very common and can be seen in the liver, mesentery, and omentum. Adjuvant therapy with tyrosine kinase inhibitors is recommended in those patients with high-risk primary GISTs.

KEYWORDS: CD117, treatment, prognosis, gastrointestinal stromal tumors, Tyrosine kinase inhibitors.

\section{INTRODUCCIÓN}

Los tumores del estroma gastrointestinal (GIST) son neoplasias raras del tracto gastrointestinal asociadas con altas tasas de transformación maligna, con una incidencia de 1/100.000 por año (1); no obstante, son los tumores mesenquimales más comunes del tracto gastrointestinal. La mayoría de los GIST se presentan de forma asintomática (2). Se identifican mejor mediante tomografía computarizada (TC) y la mayoría de las tinciones son positivas para CD117 (C-Kit), CD34 y/o DOG-1. Los datos epidemiológicos sobre los GIST en los países latinoamericanos y Ecuador son escasos, y en su mayoría corresponde a reportes de casos clínicos (3).

EI GIST es el sarcoma más común en la cavidad peritoneal y tiene diseminación metastásica. Los tumores generalmente ocurren en el estómago (55\%-60\%) y el intestino delgado (30\% $35 \%)$ y raramente en el esófago $(<1 \%)$ y el colon/recto $(5 \%)(1,2,3)$. En particular, los GIST que se encuentran en otras partes de la cavidad abdominal, generalmente en el epiplón, el mesenterio o el retroperitoneo (que representan < 5\% de todos los GIST), se conocen como tumores del tracto extra-GI (E-GIST); se considera que estos han hecho metástasis desde el estómago y/o el intestino delgado durante su desarrollo (4).

Los signos y síntomas clínicos dependen de la ubicación y el tamaño del tumor, siendo el sangrado gastrointestinal el síntoma más común, seguido de malestar abdominal, dolor, distensión abdominal y pérdida de peso (3). Los GIST pequeños, asintomáticos e indolentes se 
descubren de manera incidental, mientras que los GIST muy malignos suelen ser grandes y sintomáticos en el momento del diagnóstico (4).

El examen patológico para el diagnóstico y la evaluación del riesgo es obligatorio, y también se recomienda el genotipado para los GIST de alto riesgo. Las técnicas de resección endoscópica, aunque factibles, no están indicadas de forma rutinaria para la mayoría de los mini-GIST o micro-GIST $(1,3,4)$. Esta investigación documental tiene como objetivo proporcionar una actualización sobre los avances recientes en el diagnóstico y tratamiento de los tumores del estroma gastrointestinal.

\section{MATERIALES Y MÉTODOS}

Se realizó una búsqueda de literatura médica para conocer las características generales de los tumores del estroma gastrointestinal en bases de datos de PubMed, Elsevier y Scielo. La búsqueda incluyó articulos desde enero del 2010 hasta diciembre del 2020. Se utilizaron estrategias rastreo de información científica con los siguientes términos del vocabulario MeSH: "CD117" OR "treatment" OR "prognosis" OR "gastrointestinal stromal tumors" OR "Tyrosine kinase inhibitors"; y de su traducción al español en el vocabulario DeCS. Se aplicaron los siguientes criterios de inclusión: artículos de revisión narrativa o sistemática publicados en los últimos 10 años y realizados en seres humanos. No se realizó descarte según el idioma. El criterio de exclusión fueron artículos de estudios experimentales. Para la lectura crítica en texto completo se escogieron 15 artículos y se adicionaron 5 artículos más procedentes de las citaciones de los documentos iniciales, los cuales se consideraron relevantes para la revisión del tema.

\section{RESULTADOS}

Los tumores del estroma gastrointestinal (GIST) se consideran neoplasias potencialmente malignas que surgen de células mesenquimales, que pueden diferenciarse en las células intersticiales de Cajal, las células marcapasos del tracto GI (1). Aunque los GIST pueden surgir a cualquier edad, incluso en niños y adultos jóvenes menores de 20 años, la mayoría de los GIST se identifican en personas de mediana edad (2). Los GIST primarios rara vez invaden los tejidos circundantes. Las metástasis a los ganglios linfáticos son raras, excepto en los tipos pediátricos de GIST. Los GIST se diseminan principalmente al hígado y la cavidad peritoneal $(3,4)$.

La cirugía sigue siendo el pilar del tratamiento de los GIST primarios y es la única terapia potencialmente curativa. Aproximadamente el $60 \%$ de los pacientes con GIST clínicamente relevantes se curan únicamente con cirugía, mientras que el $40 \%$ restante de los pacientes desarrollará una enfermedad recurrente $(2,3)$. El tamaño del tumor $(\mathrm{cm})$, la mitosis (por 20 o 50 campos de alta potencia, según el tamaño del campo del microscopio o por campo de 5 $\mathrm{mm}^{2}$ ), la ubicación (gástrica o no gástrica) y la presencia o ausencia de rotura tumoral son factores de pronóstico independientes después de la resección completa (4).

Varios informes han confirmado GIST que miden $<2 \mathrm{~cm}$ (GIST por debajo de $2 \mathrm{~cm}$ ) tanto en el estómago como en el intestino. Estos incluyen "mini-GIST" $(1-2 \mathrm{~cm})$ y “micro-GIST" $(<1 \mathrm{~cm})$. LoS datos sobre la historia natural de estos GIST son mínimos y su importancia diagnóstica y la estrategia de tratamiento óptima no están claros, incluso en varias guías internacionales $(3,4)$. 


\section{Epidemiología}

Los GIST son raros y representan del $1 \%$ al $2 \%$ de las neoplasias gastrointestinales. Los GIST, tienen un comportamiento biológico variable, suelen aparecer en personas de edad avanzada (edad, 55-65 años; mediana de edad, 63 años) y rara vez se observan en personas jóvenes menores de 20 años $(0,4 \%)(1,3,4)$.

Un estudio realizado por Mullady D, en el 2016, revisaron 29 estudios que consistían en 13,550 pacientes de 19 países diferentes con GIST entre enero de 2000 y diciembre de 2014 (5). La mediana de edad fue de 65 años (rango, 10-100) con una proporción de hombres y mujeres de 1: 1. Hong Kong, Shanghai, Taiwán y Noruega reporta las tasas de incidencia más elevadas (1922 por millón por año) (6). Los GIST que ocurren fuera del estómago están asociados con un mayor potencial maligno. El crecimiento exofítico se observa en el 79\% de los GIST mientras que el intraluminal o el crecimiento mixto ocurre con menos frecuencia (7).

\section{Patología e inmunohistoquímica}

Los GIST primarios suelen estar bien delimitados, son blandos, friables y, a menudo, de naturaleza muy vascular (1). Frecuentemente parecen heterogéneos debido a necrosis o hemorragia intratumoral. Los tumores grandes pueden mostrar ulceración de la mucosa suprayacente. Microscópicamente, la mayoría de los GIST muestran tres subtipos histológicos principales: tipo de células fusiformes (el más común 70\%), tipo epitelioide (20\%), tipo mixto (10\%) (5). Más del $95 \%$ de los GIST son positivos para la proteína receptora de tirosina quinasa $(\mathrm{KIT})$, que es detectada por el anticuerpo CD117. Otros marcadores comunes son CD34 $60 \%$ e70\%) y actina de músculo liso (AML) (30\% e40\%). Por lo general, son negativos para la desmina y la proteína $\mathrm{S}-100$ ( $<5 \%$ de positivos). En contraste, los leiomiomas y leiomiosarcomas son positivos para AML y desmina, y negativos para KIT y CD34, lo que ayuda a distinguir los GIST de otros tumores mesenquimales $(6,7)$.

\section{Fisiopatología}

Los GIST son típicamente (70\%) tumores benignos, que se producen cuando los genes que controlan la expresión de la tirosina quinasa sufren una mutación que conduce a un crecimiento neoplásico que involucra células del linaje de las células intersticiales de Cajal (1). Los GIST ocurren con mayor frecuencia a lo largo del tracto gastrointestinal, pero pueden ocurrir extra gastrointestinal en casos raros (3). Los hallazgos más comunes de GIST incluyen lesiones subendoteliales que se observan en una endoscopia o una masa realzada con contraste con un margen liso en la TC. Para los GIST malignos, la diseminación linfática es extremadamente rara y estas lesiones se diseminan con mayor frecuencia por vía hematógena (más comúnmente al hígado) o al peritoneo (7).

\section{Manifestaciones clínicas}

La presentación clínica de los pacientes con GIST varía según la ubicación anatómica del tumor, el tamaño del tumor y su agresividad. Los GIST son masas submucosas muy vasculares y típicamente crecen hacia afuera, lejos de la luz GI de origen (4). Los tumores pequeños $(<2 \mathrm{~cm})$ se encuentran con mayor frecuencia de manera incidental durante la endoscopia, imágenes de corte transversal o en laparotomía/laparoscopia para otras indicaciones (5). El hallazgo accidental puede provocar un retraso significativo en el diagnóstico. Actualmente, un número importante de pacientes presenta metástasis en el momento del diagnóstico (hasta un $50 \%$ en algunas series). La mayoría (70\%) de los pacientes diagnosticados con GIST tienen síntomas 
vagos, como dolor abdominal, hemorragia digestiva por erosión de la mucosa o una masa abdominal (8).

Las manifestaciones clínicas dependen de la ubicación del tumor primario. No hay diferencia entre el sexo y la edad media informada es aproximadamente de 60 a 70 años. Por lo general, estos tumores se asocian con síntomas inespecíficos (saciedad temprana, hinchazón) a menos que se ulceren, sangren o crezcan lo suficiente como para causar dolor, obstruir o presentar otras manifestaciones relacionadas con su tamaño desproporcionado (8). En el caso del GIST esofágico, la disfagia representa el primer síntoma específico en esta localización.

En términos generales, la manifestación más frecuente es el sangrado gastrointestinal, evidente $u$ oculto, que puede estar asociado a anemia $y$, en ocasiones, a melena o hematemesis; el sangrado es el síntoma más frecuente en el caso de los GIST de intestino delgado y, a menudo, requiere una intervención quirúrgica urgente $(1,4)$. Debido a que sus tumores de crecimiento silencioso pueden ser particularmente grandes y causar distensión abdominal o una masa palpable y, en ocasiones, provocar obstrucción intestinal ( $25 \%-40 \%)$; sin embargo, rara vez se ha descrito perforación intestinal (8).

Los síndromes paraneoplásicos son inusuales en el caso de GIST; sin embargo, algunos han sido reportados como hipotiroidismo tísico o hipoglucemia secundaria a la producción de IGF-II, por lo que deben incluirse en el diagnóstico diferencial cuando aparecen síntomas endocrinometabólicos. Los pacientes con enfermedad multifocal generalmente se clasifican en estadios avanzados (metastásicos), pero debe tenerse en cuenta, particularmente en aquellos casos con afecciones hereditarias, que pueden ser posibles múltiples primarios (8).

\section{Métodos de diagnóstico}

La tomografía computarizada (TC) es el estándar de oro para la obtención de imágenes que se utiliza para caracterizar cualquier masa abdominal además de evaluar su extensión y la presencia/ausencia de enfermedad a distancia (los GIST hacen metástasis con mayor frecuencia al hígado, epiplón y cavidad peritoneal) (8). Por tanto, ante la sospecha de un tumor en el tracto digestivo, se debe realizar una TC inicial. Cabe señalar que, para el rendimiento óptimo de la TC, se debe administrar contraste oral e intravenoso para definir los márgenes intestinales (9). Las imágenes características habituales que se observan en estas imágenes incluyen la presencia de una masa sólida de contorno suave que se realza con contraste intravenoso en el caso de la TC. Los tumores muy grandes pueden parecer más complejos debido a necrosis, hemorragia o componentes degenerativos, y puede ser difícil identificar el origen de una gran masa debido al crecimiento exofítico (10).

La resonancia magnética (RM) tiene un rendimiento diagnóstico comparable al de la TC y la ventaja de carecer de radiación ionizante; sin embargo, la TC es el estudio de imagen inicial preferido para la detección y estadificación de la enfermedad (8). Hay excepciones a este proceso; por ejemplo, hay pacientes que no pueden recibir contraste intravenoso por diversos motivos (alergias). Además, la resonancia magnética a veces puede ser la opción para los GIST que se encuentran en ubicaciones específicas (como el recto) y es especialmente útil para 
evaluar el grado anatómico de la cirugía o para evaluar la sospecha de metástasis hepáticas $(9,10)$.

En presencia de una masa gástrica, está indicada la endoscopia para caracterizar la lesión (4). La endoscopia simple carece de la capacidad de distinguir con precisión entre tumores intramurales y extramurales $(9,10)$. En este sentido, la ecografía endoscópica (ECE) ha demostrado ser una técnica valiosa, pudiendo caracterizar tales masas identificando la capa de origen y permitiendo la adquisición de tejido mediante punción guiada para estudios de diagnóstico anatomopatológico, lo cual es adecuado para pruebas inmunohistoquímicas (11). Por ECE, los GIST son típicamente hipoecoicos y se presentan como lesiones homogéneas con márgenes bien definidos, aunque hay un pequeño número de casos tumorales descritos de tumores que pueden tener márgenes irregulares y ulceraciones.

\section{Tratamiento quirúrgico}

La cirugía es el único tratamiento potencialmente curativo para la sospecha de GIST resecable. El objetivo principal de este proceso es asegurar que se obtengan márgenes de resección claros en una resección completa del tumor y que se pueda extirpar sin rotura de la pseudocápsula tumoral (2); sin embargo, los márgenes amplios no tienen ningún beneficio en el control de la enfermedad. La cirugía conservadora debe ser el procedimiento de elección debido al comportamiento infiltrativo local de GIST (4); la linfadenectomía no es necesaria debido a la afectación linfática, que es rara. Es importante realizar una exploración minuciosa del hígado y del peritoneo parietal para objetivar posibles metástasis (11).

Al menos el $40 \%-50 \%$ de los pacientes que se han sometido a una cirugía óptima pueden experimentar una recurrencia del tumor; sin embargo, con la aparición de TKI, se ha ofrecido una nueva opción de control de la enfermedad. El tratamiento de los GIST $<2 \mathrm{~cm}$ es controvertido; a pesar de que un seguimiento activo de la lesión podría ser una opción, se debe considerar la cirugía porque no existen datos sobre el comportamiento de crecimiento y el potencial metastásico (11).

\section{Tratamiento coadyuvante}

El resultado de la cirugía sola ha sido inadecuado, con hasta el $50 \%$ de los pacientes que desarrollan recidiva tumoral local o distante, con una mediana de tiempo hasta la recidiva de 2 años y finalmente mueren a causa de la enfermedad. Los GIST notoriamente no responden a la quimioterapia y la radioterapia (8). El uso de TKI contra GIST introdujo una nueva era en terapias moleculares dirigidas en oncología clínica. El oncólogo juega un papel importante en el tratamiento de GIST para realizar la indicación del TKI para tumores metastásicos después de una cirugía curativa o como tratamiento neoadyuvante (9). Casi el $85 \%$ de los GIST tienen una mutación en KIT o del factor de crecimiento derivado de plaquetas (FCDP) que induce la activación de KIT, que es un receptor de tirosina quinasa que estimula el crecimiento de células cancerosas. Los tratamientos aprobados por la FDA son imatinib, sunitinib y regorafenib (12).

\section{Pronóstico y recurrencia}


El mantenimiento de la normoxia tisular, la reversión de la hipoxia tisular y la evitación de la disoxia tisular son esenciales para prevenir la deuda de oxígeno, lesión celular, insuficiencia orgánica y la muerte (2). Los marcadores de extracción y perfusión de oxígeno definen la presencia de enfermedad, cuantifican la gravedad, proporcionan una hoja de ruta clínica para guiar las intervenciones y proporcionar pronóstico (3). La capacidad de caracterizar a los pacientes según un fenotipo hemodinámico distinto reduce la heterogeneidad de los pacientes y aclara la selección de pacientes y los resultados de los ensayos de resultados relacionados. Al controlar estos factores, se puede mejorar la fidelidad de la señal de los ensayos de resultados de la intervención. El pronóstico de los pacientes con GIST se basa en varias características, principalmente: sitio del tumor primario, recuento mitótico y tamaño del tumor. Otros factores que contribuyen al pronóstico de los GIST incluyen la presencia de márgenes negativos y evitar la rotura del tumor en la resección (12).

En 2001, los Institutos Nacionales de Salud (NIH) de EE. UU, celebraron una conferencia de expertos con el objetivo de desarrollar un enfoque de consenso para el diagnóstico y pronóstico morfológico de GIST. Un esquema para predecir el riesgo de recurrencia o metástasis de un GIST primario resecado quirúrgicamente fue desarrollado por consenso en esta reunión y fue publicado por Fletcher et al, en el $2002(3,12)$. El tamaño del tumor y el índice mitótico fueron la base de este enfoque de consenso para la estratificación del riesgo de los GIST. En general, los tumores $<5 \mathrm{~cm}$ y particularmente los $<2 \mathrm{~cm}$ tienen un menor riesgo de metástasis, mientras que los tumores $>5 \mathrm{~cm}$ y particularmente los $>10 \mathrm{~cm}$ tienen un riesgo mayor (13). De manera similar, una tasa mitótica de $<5$ mitosis por 50 campos de alto poder (CAP) presagia un bajo riesgo de metástasis, mientras que las tasas mitóticas $>5$ por 50 CAP y particularmente aquellas $>10$ por 50 CAP predicen un mayor riesgo de enfermedad metastásica. Estos 2 factores son predictores independientes, pero mutuamente influyentes y se combinan en las directrices de los NIH (14).

\section{DISCUSIÓN}

El tumor del estroma gastrointestinal es una entidad clínica rara pero importante que se observa en la práctica clínica. Aunque no se conoce la prevalencia exacta de GIST, la incidencia de GIST ha ido en aumento (3). La mayoría de los GIST ocurren debido a una mutación en el gen c-kit o en el gen del receptor alfa del factor de crecimiento derivado de plaquetas. El 15\% de los GIST no tienen estas mutaciones y se denominan GIST de tipo salvaje. Casi todos los GIST expresan la tirosina quinasa del receptor KIT (10). La agresividad de los GIST depende del tamaño, el índice mitótico y la ubicación. La resección quirúrgica es el tratamiento de elección. Pero cada vez se prueban más diversas modalidades endoscópicas de resección (15).

La resección quirúrgica sigue siendo la piedra angular del GIST primario temprano y localmente avanzado o recurrente. La integración y la sincronización de los inhibidores de la tirosina quinasa sistémica es el factor decisivo adicional para la supervivencia general y libre de recurrencia. La extirpación quirúrgica con escisión completa y sin ruptura del tumor es la única terapia curativa y pilar para los GIST resecables primarios localizados (15).

La mayoría de los GIST surgen de la muscularis propia del estómago o de la pared intestinal y son de crecimiento exofítico, lo que da como resultado una protrusión del tejido de origen y 
explica el hecho de que los márgenes quirúrgicos negativos de resección son relativamente fáciles de obtener (16). El margen microscópico de resección no se correlaciona con la supervivencia y no se considera tan importante como si las células tumorales se transfieren directamente a la cavidad peritoneal, aunque en el estudio de Ricci et al (17), prohíbe una conclusión final. Por esa razón, las resecciones en cuña o resecciones segmentarias del órgano subyacente se utilizan comúnmente para tratar los GIST primarios y Ortenzi M (15), incluso informaron que la resección amplia no tenía ningún beneficio conocido.

Debido a la fragilidad del tumor, puede presentarse complicaciones. En consecuencia, es imprescindible una técnica quirúrgica detallada y cuidadosa para evitar la rotura del tumor intraoperatoria, que es un indicador de mal pronóstico. Thacoor A (11), concluye que la rotura del tumor redujo la mediana de supervivencia de 46 a 17 meses, una cifra comparable a la mediana de supervivencia después de una resección incompleta (21 meses). Patel $N(14)$, informó una mediana de supervivencia para los pacientes que se sometieron a una resección completa de 46 meses en comparación con 10 meses para los que se sometieron a una resección incompleta.

Askari A (13), analizaron el pronóstico después de la resección primaria de GIST y concluyeron que los GIST de bajo grado tenían un pronóstico excelente después de la resección sola. Sin embargo, se encontró que los GIST de alto grado se asociaron con un alto riesgo de recurrencia después de la resección primaria. Thacoor A (11), también identificó factores de pronóstico desfavorables como resección incompleta, características histológicas de alto grado y tamaño del tumor de $5 \mathrm{~cm}$ o más $(p<0,5)$. Además, se encontraron que el grado del tumor es el predictor más importante en pacientes con resección completa (mediana de supervivencia, 55 frente a 19 meses en 35 pacientes; $p<0,05)$.

La resección quirúrgica completa aún puede ir seguida de recurrencia. Se ha informado que los pacientes que poseían la mutación del exón 11 mostraron tasas más altas de recurrencia. Nishida T (2) y Barnett C (7)afirmaron que no había ningún beneficio de la detección temprana de GIST recurrentes para mejorar la supervivencia. En el Roswell Park Cancer Institute, se informaron tasas de recurrencia del $76 \%$ para los tumores gástricos y del $64 \%$ para los tumores del intestino delgado y grueso. La recurrencia se observó en el $80 \%$ de los pacientes durante el seguimiento después de la resección completa en un estudio de $\mathrm{Ng}$ et al. El Centro de Cáncer Memorial Sloan-Kettering reportó en 60 pacientes, la recurrencia local del $76 \%$ de los pacientes (18).

\section{CONCLUSIONES}

Los GIST son tumores poco frecuentes que representan un pequeño porcentaje de neoplasias gastrointestinales. Los GIST que ocurren fuera del estómago están asociados con un mayor potencial de malignidad. Por lo general, los GIST son un hallazgo incidental y, por lo tanto, la mayoría de las veces se presentan de forma asintomática. La patología de los GIST consiste en células fusiformes, células epitelioides o tipos de células mixtas. Los GIST suelen ser positivos para CD117 y DOG-1. 
La resección quirúrgica laparoscópica de GIST con imatinib adyuvante $400 \mathrm{mg}$ al día es el estándar de oro para el tratamiento de GIST. Sin embargo, si el tumor es irresecable, se recomienda imatinib neoadyuvante $400 \mathrm{mg}$ al día seguido de resección. Las metástasis son muy comunes y se pueden ver en el hígado, el mesenterio y el epiplón, pero se tratan de la misma manera que los GIST de alto riesgo.

El riesgo de malignidad potencial y recurrencia, se determina con las clasificaciones NIH, AFIP y NIH modificadas que se calculan en función del tamaño del tumor, la tasa mitótica, la ubicación y la perforación. Es fundamental que los médicos incluyan los GIST en el diagnóstico diferencial debido a la importancia del diagnóstico precoz en estos casos.

Se recomienda el tratamiento coadyuvante en aquellos pacientes que tienen GIST primarios de alto riesgo R0; sin embargo, la indicación óptima de tratamiento adyuvante en pacientes de alto riesgo no está clara, por lo que cada caso debe abordarse de forma individual en comités multidisciplinares especializados que equilibren los impactos beneficiosos y negativos. Los inhibidores de la tirosina quinasa se recomiendan para los GIST de alto riesgo.

La última década marcó una era importante en la historia de tumores GIST, que culminó desde el avance del diagnóstico de GIST y la comprensión de su patogénesis, hasta el desarrollo de puntuaciones de pronóstico de riesgo $y$, en consecuencia, influyendo en las estrategias de tratamiento. Esta traducción de los éxitos de laboratorio en terapias biológicamente relevantes mejora drásticamente los resultados de los pacientes. Los estudios futuros deben centrarse en cómo integrar bien la terapia molecular con la cirugía para el tratamiento de GIST operables o inoperables. Ahora se necesitan grandes ensayos clínicos multiinstitucionales para investigar la eficacia de los inhibidores de la tirosina quinasa como terapia adyuvante o neoadyuvante para los GIST.

Los GIST son el paradigma de un cáncer con terapia molecular dirigida y su manejo requiere un enfoque multidisciplinario.

\section{REFERENCIAS}

1. Sorour M. Gastrointestinal stromal tumors (GIST) related emergencies. Int J Surg. Vol.12(4):269-80. doi: 10.1016/j.ijsu.2014.02.004.. 2014.

2. Nishida T. The standard diagnosis, treatment, and follow-up of gastrointestinal stromal tumors based on guidelines. Gastric Cancer. Vol.19(1):3-14. doi: 10.1007/s10120-0150526-8.. 2016.

3. Ahmed M. Recent advances in the management of gastrointestinal stromal tumor. World J Clin Cases. Vol.8(15):3142-3155. doi:10.12998/wjcc.v8.i15.3142. 2020.

4. Valsangkar N. Current management of gastrointestinal stromal tumors: Surgery, current biomarkers, mutations, and therapy. Surgery. Vol.158(5):1149-64. doi: 10.1016/j.surg.2015.06.027. 2015.

5. Mullady D. A multidisciplinary approach to the diagnosis and treatment of gastrointestinal stromal tumor. J Clin Gastroenterol. Vol.47(7):578-85. doi: 10.1097/MCG.0b013e3182936c87. 2016. 
6. Grupo Español de Investigación en Sarcomas/Spanish Group for Sarcoma Research. GEIS guidelines for gastrointestinal sarcomas (GIST). Cancer Treat Rev. Vol.55:107-119. doi: 10.1016/j.ctrv.2016.11.011.. 2017.

7. Barnett C. Gastrointestinal stromal tumors: molecular markers and genetic subtypes. Hematol Oncol Clin North Am. Vol.27(5):871-88. doi: 10.1016/j.hoc.2013.07.003. 2013.

8. Burch J. Gastrointestinal Stromal Cancer. [Updated 2020 Jun 9]. In: StatPearls [Internet]. Treasure Island (FL): StatPearls Publishing; 2020 Jan-. Available from: https://www.ncbi.nlm.nih.gov/books/NBK554541/. 2020.

9. Rare Tumors Gi Group. Comprehensive review into the challenges of gastrointestinal tumors in the Gulf and Levant countries. World J Clin Cases. Vol.8(3):487-503. doi:10.12998/wjcc.v8.i3.487. 2020.

10. Reichardt P. Gastrointestinal stromal tumors: evolving role of the multidisciplinary team approach in management. Expert Rev Anticancer Ther. Vol.12(8):1053-68. doi: 10.1586/era.12.48.. 2012.

11. Thacoor A. Gastrointestinal stromal tumours: advances in surgical and pharmacological management options. J Gastrointest Oncol. Vol.9(3):573-578. doi:10.21037/jgo.2018.01.10. 2018.

12. Parab et al. Gastrointestinal stromal tumors: a comprehensive review. J Gastrointest Oncol. Vol.10(1):144-154. doi:10.21037/jgo.2018.08.20. 2019.

13. Askari A. Unusual presentations, management and outcomes of gastric stromal tumors: a single-center case series. Ann Gastroenterol. Vol.34(1):26-32. doi:10.20524/aog.2020.0540. 2020.

14. Patel N. Incidence of Gastrointestinal Stromal Tumors in the United States from 20012015: A United States Cancer Statistics Analysis of 50 States. Cureus. Vol.11(2):e4120. doi:10.7759/cureus.4120. 2019.

15. Ortenzi M. Surgical treatment of gastric stromal tumors: laparoscopic versus open approach. Ann Ital Chir. Vol.88:S0003469X17026112. 2017.

16. Rajendra R. Management of gastrointestinal stromal tumors. Future Oncol. Vol.9(2):193206. doi: 10.2217/fon.12.178. 2013.

17. Ricci R. Syndromic gastrointestinal stromal tumors. Hered Cancer Clin Pract. Vol.14:15. doi: 10.1186/s13053-016-0055-4. 2016.

18. Sanchez-Hidalgo et al. Gastrointestinal stromal tumors: A multidisciplinary challenge. World J Gastroenterol. Vol.24(18):1925-1941. doi:10.3748/wjg.v24.i18.1925. 2018. 